\section{Case Reports in Ophthalmology}

\title{
Intravenous Drug Use-Associated Scopulariopsis Endophthalmitis Treated with Systemic and Intravitreal Voriconazole
}

\author{
Joseph J. Raevis Neha Shaik Joseph Tseng \\ Department of Ophthalmology, SUNY Downstate Medical Center, Brooklyn, NY, USA
}

\section{Keywords}

Endogenous · Endophthalmitis · Fungal · Intravenous drug abuse $\cdot$ Scopulariopsis

\begin{abstract}
Purpose: To report a case of intravenous (i.v.) heroin use-associated endogenous endophthalmitis caused by Scopulariopsis fungal species, and its response to intravitreal and oral voriconazole treatments. Patient: A 21-year-old-female with chronic hepatitis C and i.v. heroin use presented with subacute decreased vision to hand motion in her left eye. Results: Endogenous fungal endophthalmitis caused by Scopulariopsis was confirmed by vitreous biopsy. The patient improved clinically after vitrectomy with intravitreal voriconazole and 3 weeks of oral voriconazole. The final vision was 20/60 after 6 months. Conclusions: Scopulariopsis is a rare cause of endophthalmitis, and is often difficult to treat due to its resistance to commonly used antifungals. This case is the first report of Scopulariopsis endophthalmitis secondary to i.v. drug use.

(C) 2018 The Author(s)

Published by S. Karger AG, Basel
\end{abstract}

\section{Introduction}

Scopulariopsis, a genus of anamorphic fungi typically found in soil and decaying wood, is an opportunistic mold pathologic to humans [1]. The spectrum of infections includes pulmo- 
nary fungal balls, endocarditis, and disseminated skin lesions. Reported ocular infections include keratitis [2] and posttraumatic endophthalmitis [3, 4].

Voriconazole is one of the main intravitreal and systemic medications used to treat Scopulariopsis. It has not been shown to cause retinal toxicity with doses less than $25 \mathrm{ug} / \mathrm{mL}$ in rats [5] and has safely been used in humans orally and intravitreally. This contrasts with amphotericin B, which is used less often systemically due to its nephrotoxicity [6]. Some clinicians prefer using systemic caspofungin in addition to voriconazole in the treatment of fungal endophthalmitis [7].

Scopulariopsis endophthalmitis has been reported only with trauma and ocular postoperative patients [3, 4]. Our patient developed Scopulariopsis endophthalmitis due to intravenous (i.v.) heroin use, making this the first such case reported.

\section{Case Report}

A 21-year-old female with a history of i.v. heroin abuse and bipolar disorder presented with painless vision loss of the left eye over 4 weeks. She endorsed i.v. heroin use within weeks of the onset of visual symptoms. Examination revealed count-fingers vision in the affected left eye with trace cell in the anterior chamber. Fundus examination of the left eye revealed optic nerve head edema and vitreous debris in a "string of pearls" formation overlying a white chorioretinal lesion in the foveal center (Fig. 1b). Clinical examination of the fellow right eye was unremarkable (Fig. 1a). Preoperative fluorescein angiography of the left eye showed a lesion with early hyperfluorescence and late staining (Fig. 2). Optical coherence tomography (OCT) of the left macula preoperatively showed a foveal lesion with resultant distortion of the foveal contour, associated epiretinal membrane, and overlying vitreous inflammation (Fig. 3). The patient was diagnosed with presumed infectious fungal endophthalmitis. A vitrectomy and membrane peeling was performed, with an undiluted vitreous biopsy sample being plated on aerobic, anaerobic, and Sabouraud agar. The Sabouraud mycological culture revealed heavy growth of Scopulariopsis within 3 days of the sample being sent to the lab. Sensitivities were unable to be obtained due to the small volume of the vitreous biopsy.

The patient was admitted to the hospital and started on oral voriconazole $4 \mathrm{mg} / \mathrm{kg}$ twice a day for 3 weeks. She also received $0.1 \mathrm{~mL}$ intravitreal voriconazole intraoperatively, but refused further intravitreal treatment. Systemic workup revealed asymptomatic hepatitis C, and an echocardiogram showed no signs of vegetation. Two sets of blood cultures taken at different times were negative. One week postoperatively, the vision improved to 20/400 with reduction in the size of the central chorioretinal lesion and the optic nerve edema (Fig. 1d). The OCT showed disruption of foveal ellipsoid layer but with resolution of vitreous inflammation and no residual retinal membrane (Fig. 3). After 6 months, the OCT depicted significant improvement in the foveal contour with a vision of 20/60 (Fig. 3).

\section{Discussion}

We describe the first case of i.v. drug-associated endogenous endophthalmitis due to Scopulariopsis, the source of which is likely from contaminated needles or mixing agents during illicit drug administration. The diagnosis of mycotic endophthalmitis can be difficult due to its indolent course and sometimes unclear history. Endogenous endophthalmitis re- 


\section{Case Reports in Ophthalmology}

lated to drug use can be suspected in patients with needle tracks and skin popping [8], while blood and urine tests can help determine which patients have used recreational drugs in the more recent past. Other diagnoses, including infectious and inflammatory causes, should always be considered, and a thorough uveitis evaluation with the involvement of internal medicine and infectious disease specialists should be considered. Diagnostic studies such as echocardiography or radiographic imaging to evaluate for cardiac and pulmonary lesions may also be considered.

The visual prognosis for patients with endogenous endophthalmitis is guarded, with final visual acuities commonly reported to be 20/400 [9]. The vitreous biopsy demonstrated Scopulariopsis as the causative organism; sensitivities were not successfully obtained by the testing laboratory. However, our patient did show improvement with intravitreal and oral voriconazole therapy. While the most effective treatment for Scopulariopsis infections has yet to be elucidated, we believe that intravitreal voriconazole and systemic voriconazole ( $4 \mathrm{mg} / \mathrm{kg}$ twice daily) for 2-3 weeks may be an acceptable treatment regimen unless biopsyguided sensitivities offer other options.

\section{Statement of Ethics}

The authors of this study declare no ethical conflicts.

\section{Disclosure Statement}

The authors of this study have no conflicts of interest or proprietary interests.

\section{References}

1 Issakainen J, Heikkilä H, Vainio E, Koukila-Kähkölä P, et al: Occurrence of Scopulariopsis and Scedosporium in nails and keratinous skin. A 5-year retrospective multi-center study. Med Mycol 2007;45:201-229.

2 Malecha MA: Fungal keratitis caused by Scopulariopsis brevicaulis treated successfully with natamycin. Cornea 2004;23:201-203.

-3 Gariano RF, Kalina RE: Posttraumatic fungal endophthalmitis resulting from Scopulariopsis brevicaulis. Retina 1997;17:256-258.

4 Aydin S, Ertugrul B, Gultekin B, Uyar G, Kir E: Treatment of two postoperative endophthalmitis cases due to Aspergillus flavus and Scopulariopsis spp. with local and systemic antifungal therapy. BMC Infect Dis 2007;7:87. Doi 10.1186/1471-2334-7-87

5 Gao H, Pennesi M, Shah K, Qiao X, et al: Safety of intravitreal voriconazole: electroretinographic and histopathologic studies. Trans Am Ophthalmol Soc 2003;101:183-189.

6 Fanos V, Cataldi L: Amphotericin B-induced nephrotoxicity: a review. J Chemother 2013;12:463-470.

-7 Breit SM, Hariprasad SM, Mieler WF, Shah GK, Mills MD, Grand MG: Management of endogenous fungal endophthalmitis with voriconazole and caspofungin. Am J Ophthalmol 2005;139:135-140.

-8 Canales M, Gerhard J, Younce E: Lower extremity manifestations of "skin-popping" an illicit drug use technique: a report of two cases. Foot (Edinb) 2015;25:114-119.

-9 Patel SN, Rescigno RJ, Zarbin MA, Langer P, Bhagat N: Endogenous endophthalmitis associated with intravenous drug abuse. Retina 2014;34:1460-1465. 


\section{Case Reports in Ophthalmology}

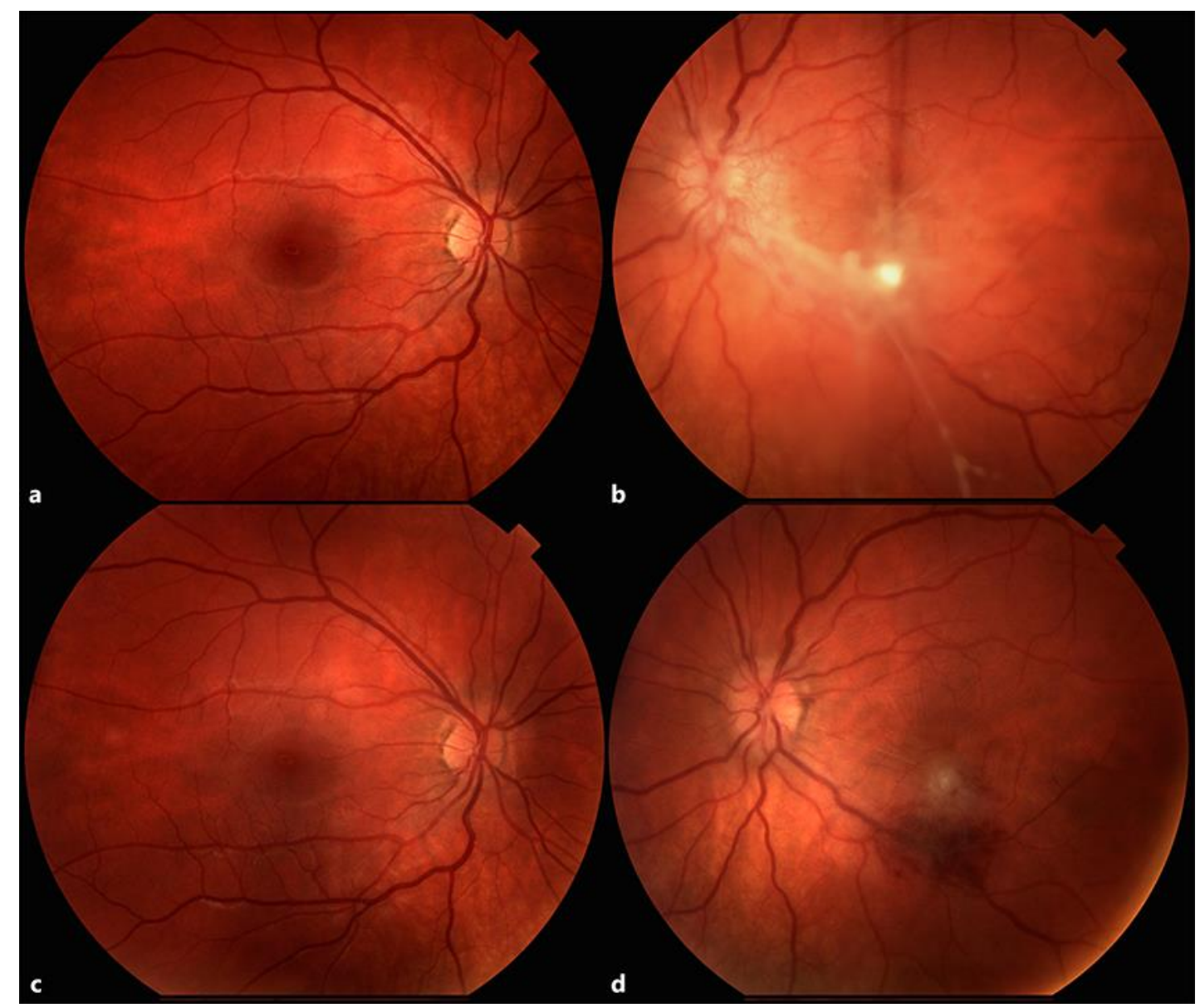

Fig. 1. Color fundus photographs of the right and left eyes preoperatively and postoperatively. Normal preoperative right fundus (a) and postoperative (c). b The left eye had optic disc edema and "string of pearls" vitreous debris from the disc to the macular overlying a white chorioretinal lesion. $\mathbf{d}$ One week after pars plana vitrectomy with intraoperative voriconazole, the disc edema appeared improved and the chorioretinal lesion relatively consolidated.

Raevis et al.: Intravenous Drug Use-Associated Scopulariopsis Endophthalmitis Treated with Systemic and Intravitreal Voriconazole 
Case Reports in
Ophthalmology

Case Rep Ophthalmol 2018;9:37-42

DOI: $10.1159 / 000485552$

(c)

2018 The Author(s). Published by S. Karger AG, Basel

Raevis et al.: Intravenous Drug Use-Associated Scopulariopsis Endophthalmitis Treated with Systemic and Intravitreal Voriconazole

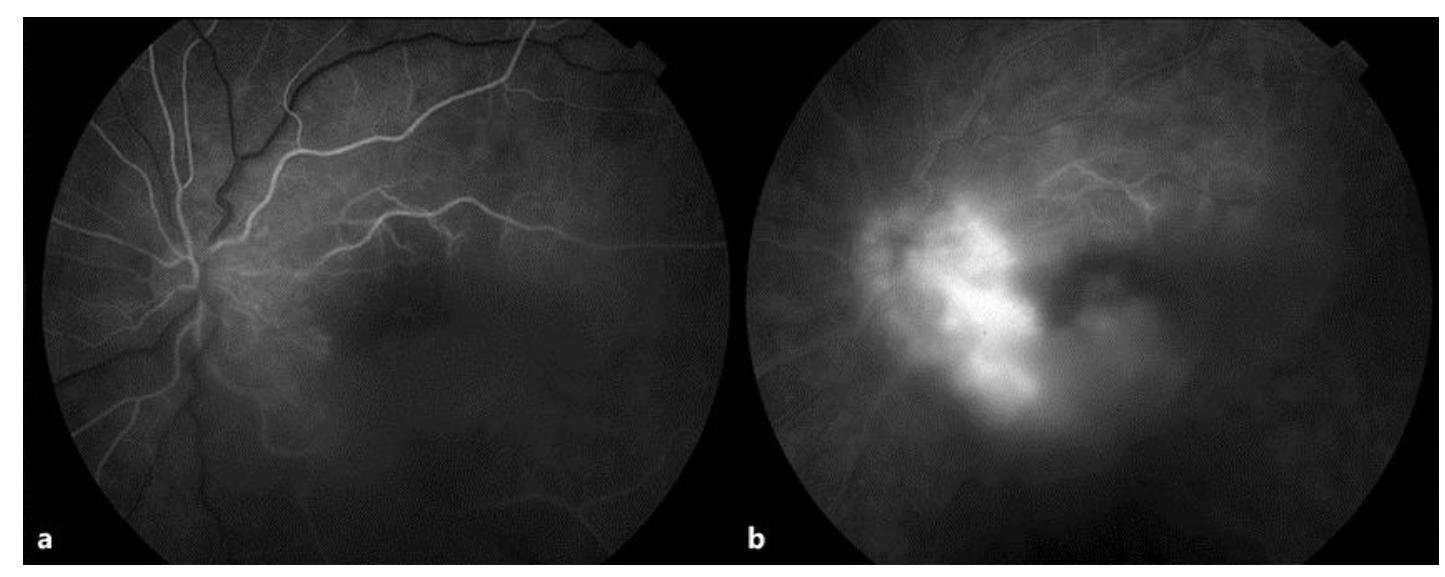

Fig. 2. Preoperative fluorescein angiography of the left eye displayed an early hyperfluorescence lesion (a) with late staining of the chorioretinal lesion (b). 


\section{Case Reports in Ophthalmology}

\begin{tabular}{l|l}
\hline Case Rep Ophthalmol 2018;9:37-42 \\
\hline DOI: $10.1159 / 000485552$ & $\begin{array}{l}\text { C 2018 The Author(s). Published by S. Karger AG, Basel } \\
\text { www.karger.com/cop }\end{array}$ \\
\hline
\end{tabular}

Raevis et al.: Intravenous Drug Use-Associated Scopulariopsis Endophthalmitis Treated with Systemic and Intravitreal Voriconazole

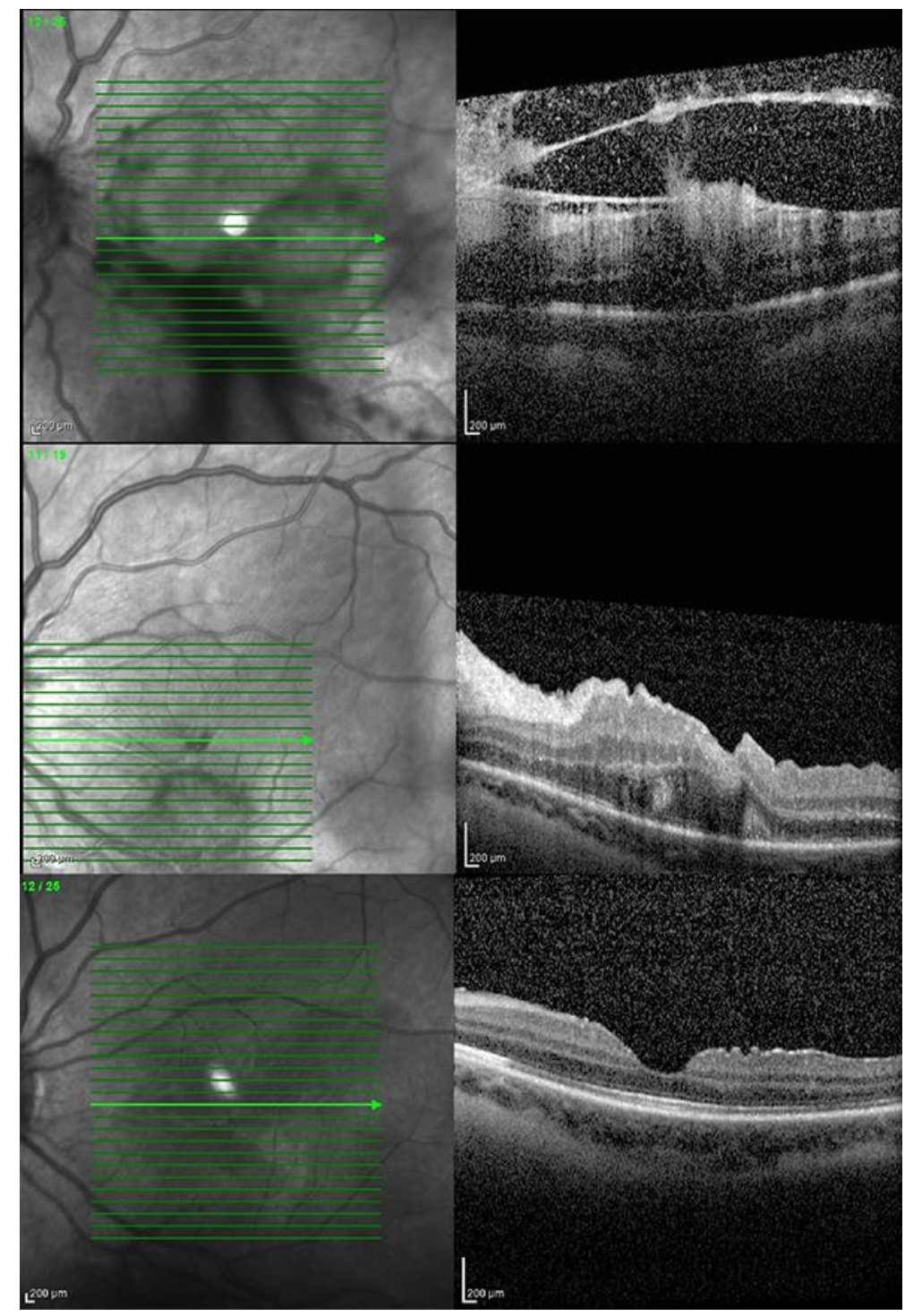

Fig. 3. Optical coherence tomography (OCT) of the left macula preoperatively showing focal vitreous inflammation with foveal contour distortion and epiretinal membrane (top). One week postoperative OCT macula showing loss of foveal ellipsoid layer but with resolution of vitreous inflammation and epiretinal membrane (middle). OCT macula at 6 months after vitrectomy depicting significant improvement in foveal contour (bottom). 